\title{
Targeted physiotherapy for patellofemoral joint osteoarthritis: A protocol for a randomised, single-blind controlled trial
} Kay M Crossley*1,2, Bill Vicenzino ${ }^{3}$, Marcus G Pandy ${ }^{1}$, Anthony G Schache ${ }^{1}$ and Rana S Hinman ${ }^{4}$

Address: ${ }^{1}$ Department of Mechanical Engineering, Melbourne School of Engineering, The University of Melbourne, Australia, ${ }^{2}$ School of Physiotherapy, The University of Melbourne, Melbourne, Australia, ${ }^{3}$ Division of Physiotherapy, School of Health and Rehabilitation Sciences, University of Queensland, Brisbane, Australia and ${ }^{4}$ Centre for Health, Exercise and Sports Medicine, School of Physiotherapy, The University of Melbourne, Melbourne, Australia

Email: Kay M Crossley* - k.crossley@unimelb.edu.au; Bill Vicenzino - b.vicenzino@uq.edu.au; Marcus G Pandy - pandym@unimelb.edu.au; Anthony G Schache - anthonys@unimelb.edu.au; Rana S Hinman - ranash@unimelb.edu.au

* Corresponding author

Published: 16 September 2008

BMC Musculoskeletal Disorders 2008, 9:122 doi:10.1186/147I-2474-9-122
Received: 26 June 2008

Accepted: 16 September 2008

This article is available from: http://www.biomedcentral.com//47/-2474/9//22

(c) 2008 Crossley et al; licensee BioMed Central Ltd.

This is an Open Access article distributed under the terms of the Creative Commons Attribution License (http://creativecommons.org/licenses/by/2.0), which permits unrestricted use, distribution, and reproduction in any medium, provided the original work is properly cited.

\begin{abstract}
Background: The patellofemoral joint (PFJ) is one compartment of the knee that is frequently affected by osteoarthritis (OA) and is a potent source of OA symptoms. However, there is a dearth of evidence for compartment-specific treatments for PFJ OA. Therefore, this project aims to evaluate whether a physiotherapy treatment, targeted to the PFJ, results in greater improvements in pain and physical function than a physiotherapy education intervention in people with symptomatic and radiographic PFJ OA.
\end{abstract}

Methods: 90 people with PFJ OA (PFJ-specific history, signs and symptoms and radiographic evidence of PFJ OA) will be recruited from the community and randomly allocated into one of two treatments. A randomised controlled trial adhering to CONSORT guidelines will evaluate the efficacy of physiotherapy ( 8 individual sessions over 12 weeks, as well as a home exercise program 4 times/week) compared to a physiotherapist-delivered OA education control treatment (8 individual sessions over 12 weeks). Physiotherapy treatment will consist of (i) quadriceps muscle retraining; (ii) quadriceps and hip muscle strengthening; (iii) patellar taping; (iv) manual PFJ and soft tissue mobilisation; and (v) OA education. Resistance and dosage of exercises will be tailored to the participant's functional level and clinical state. Primary outcomes will be evaluated by a blinded examiner at baseline, 12 weeks and 9 months using validated and reliable pain, physical function and perceived global effect scales. All analyses will be conducted on an intention-to-treat basis using linear mixed regression models, including respective baseline scores as a covariate, subjects as a random effect, treatment condition as a fixed factor and the covariate by treatment interaction.

Conclusion: This RCT is targeting PFJ OA, an important sub-group of knee OA patients, with a specifically designed conservative intervention. The project's outcome will influence PFJ OA rehabilitation, with the potential to reduce the personal and societal burden of this increasing public health problem.

Trial Registration: Australia New Zealand Clinical Trials Registry ACTRNI 2608000288325 


\section{Background}

Osteoarthritis (OA) is the leading cause of musculoskeletal pain and disability and is the third leading cause of lifeyears lost due to disability in Australia, only behind depression and dementia [1]. The annual total cost of arthritic disease in Australia is estimated at \$24 billion [2], with the knee joint contributing substantially to this overall cost. The prevalence of OA in people aged over 55 years is $20-26 \%$ and rising, with arthritis rates expected to increase by $30 \%$ over the next 40 years [2]. The pain and suffering endured by patients as a result of OA decreases their quality of life, with the annual burden of disease costs ( $\$ 12$ billion in Australia) being half the total costs associated with this condition [2]. Pain associated with daily activities such as walking and stair-climbing ultimately leads to profoundly reduced functional independence [2].

The patellofemoral joint (PFJ) is one of the three knee joint compartments. Awareness of its importance in the OA process has been raised by the increasing use of lateral and skyline $\mathrm{x}$-rays in recent times. Research has revealed that PFJ OA is more common than previously thought. In a community-based study of knee OA $(\mathrm{N}=218)$, the frequency of radiographic osteophytes was greater in the PFJ (65\% knees) than in the tibiofemoral joint (TFJ) $(55 \%$ knees) [3]. Furthermore, in people with knee pain $(\mathrm{N}=$ 777), the most common compartmental distribution of radiographic OA was a combination of TFJ and PFJ disease (40\%), followed by isolated PFJ OA (24\%), and isolated TFJ disease (4\%) [4]. Within the PFJ, the lateral compartment is more frequently affected by the OA process than the medial $[5,6]$. Importantly, the presence of baseline PFJ OA predicts structural deterioration in the TFJ compartment over 30 months (OR 2.31, 95\% CI 1.37, 3.88) [7].

The PFJ is an important source of symptoms associated with knee OA [8]. Knee pain has been found to be significantly associated with PFJ osteophytes (OR 2.25, 95\%CI $1.06,4.77$ ), but not TFJ osteophytes (OR 1.19, 95\% CI $0.46,3.09)[9]$, suggesting that the PFJ may be a more important source of knee pain than the TFJ. Hunter et al [10] noted that increased pain and poorer function was associated with reduced cartilage volume in the patella, but not in the femur nor the tibia. Other authors have confirmed the relationship between radiographic PFJ OA and knee pain [11-13].

Management strategies for knee OA have traditionally focussed on alleviating symptoms, primarily using drug therapies or surgery. A meta-analysis of OA trials highlights this, with most trials evaluating drug treatments $(60 \%)$ or surgical procedures (26\%) [14]. OA experts have highlighted the overall dearth of quality evidence to support the use of non-pharmacological interventions such as physiotherapy. Despite this, knee OA clinical guidelines recommend that conservative treatments be included as a first line strategy for the optimal management of the disease $[15,16]$. Physiotherapy is a conservative intervention, which is non-toxic, inexpensive and promotes physical activity and self management through exercise. Therefore, rigorous randomised clinical trials (RCTs) that evaluate the efficacy of physiotherapy are clearly needed, to better guide clinical decision-making.

Given the heterogeneity of knee OA with regard to aetiology, clinical presentation and natural history, guidelines also recommend the tailoring of knee OA treatments to the location of joint damage in order to optimise treatment outcomes $[15,16]$. However, most trials of physiotherapy for knee OA have not been targeted to disease subgroups, with participant selection typically based on the presence of non-specific knee pain and radiographic changes anywhere on an anteroposterior radiograph. While a plethora of evidence attests to the benefits of exercise for patients with predominant TFJ OA [17] there is no level I evidence and only one RCT [18] specifically addressing the problem of PFJ OA. The dearth of evidence for a compartment-specific treatment for PFJ OA necessitates our proposed study to establish the efficacy of a compartment-specific physiotherapy treatment using the rigour of a RCT.

While there is little known about the physical impairments associated with PFJ OA, there are several RCTs that have evaluated physical interventions for PFJ pain in younger adults (patellofemoral pain syndrome, or anterior knee pain). We have previously conducted a double blind, placebo-controlled RCT [19], which demonstrated the efficacy of a targeted physiotherapy program for this patient population. The targeted treatment involved (i) quadriceps muscle retraining; (ii) patellar taping; (iii) manual PFJ and soft tissue mobilisation; and (iv) hip muscle retraining. We have recently confirmed the beneficial effects of this targeted physiotherapy approach on pain and physical function in another population of young adults with PFJ pain [20]. Therefore, we are proposing to evaluate a similar, targeted physiotherapy intervention for people with PFJ OA.

This project aims to evaluate whether a physiotherapy treatment, targeted to the PFJ and based on successful treatment for PFJ pain in younger populations, results in greater improvements in pain and physical function than a physiotherapy education intervention in participants with symptomatic and radiographic PFJ OA. 


\section{Methods}

\section{Experimental design}

A randomised, single-blind, controlled clinical trial conforming to CONSORT [21] guidelines will be conducted, comparing a multimodal physiotherapy intervention to a physiotherapy education intervention (Figure 1). A Project Investigator will screen for eligibility based on history, clinical and radiographic examination.

Ethical approval has been obtained from the University of Melbourne Human Research Ethics Committee (HREC No. 0721163) and from the Department of Human Services Victoria, Radiation Safety Committee. All participants will provide written informed consent.

\section{Participants}

Ninety people with lateral PFJ OA will be recruited from the community via advertisements, medical practitioners and our own research database. To be included in the study, participants must fulfil the following criteria: (i) aged > 40 years; (ii) anterior- or retro-patellar knee pain aggravated by at least two activities that load the PFJ (eg stair ambulation, squatting and/or rising from sitting); (iii) pain severity $\geq 4$ on an 11 point numerical pain scale during aggravating activities; (iv) pain during these activities present on most days during the past month; (v) osteophyte grade $\geq 1$ in the lateral PFJ compartment on skyline x-ray [22].

Exclusion criteria will include: (i) concomitant pain from other knee structures, hip or lumbar spine; (ii) current or previous physiotherapy for knee pain (prior 12 months); (iii) contra-indications to the treatments (eg tape allergy); (iv) recent knee injections (prior 3 months); (v) planned lower limb surgery in the following 9 months; (vi) body mass index $\geq 35 \mathrm{~kg} \cdot \mathrm{m}^{2}$; (vii) medial PFJ OA (osteophytes
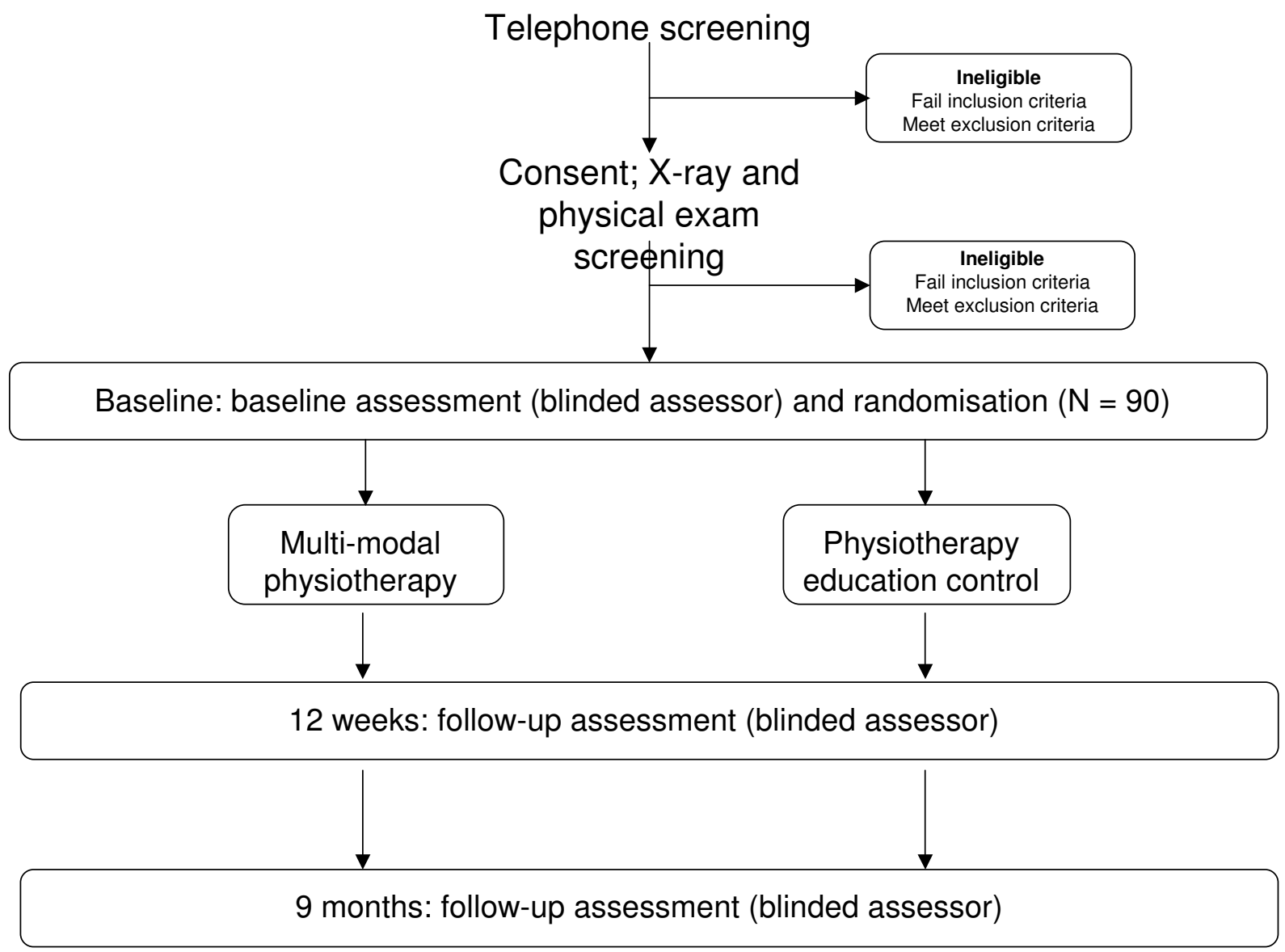

Figure I

Flow of participants through the randomised controlled trial. 
or joint space narrowing on a skyline $\mathrm{x}$-ray) that is more severe than lateral PFJ OA; (viii) moderate to severe concomitant TFJ OA (Kellgren and Lawrence grade $\geq 3$ on an anteroposterior radiograph) [23]; (ix) knee or hip arthroplasty or osteotomy; (xi) physical inability to undertake testing procedures or; $(\mathrm{x})$ inability to understand written and spoken English.

\section{Sample Size}

Treatment efficacy will be evaluated by comparing change on primary outcome measures between groups. We aim to detect the minimum clinically important improvement on these outcomes as reported by Tubach et al [24]. Specifically, a sample of 90 will provide a minimum of $90 \%$ power $(\alpha=0.05)$ to detect a difference in pain on visual analogue scale (VAS) of 19.9 (21.5) $\mathrm{mm}$ and a difference in physical function on the Western Ontario MacMaster Universities Osteoarthritis Index (WOMAC) [25] of 9.1 (13.9) normalised units. This sample size also allows for an estimated $10 \%$ drop-out rate.

\section{Procedure}

The randomisation schedule (permuted blocks of 8 to 12) will be generated and maintained centrally by one of the investigators (BV), who will not be involved in assessment of participants. The randomisation schedule will be revealed via telephone following baseline assessment. A blinded investigator will perform outcome assessments (Table 1) at baseline, 12 weeks and 9 months, and participants will be instructed not to divulge their group allocation. Security of the blinding system will be evaluated to ensure integrity.

\section{Outcome assessment}

Age, gender, duration of knee OA symptoms, previous treatment, surgery and medication use for knee OA will be obtained at the baseline assessment.

\section{Primary outcome measures: Pain and physical function}

Overall average knee pain in the previous week on movement and during an aggravating activity nominated by the participant will be self-assessed with a 0-100 $\mathrm{mm}$ horizontal visual analogue scale (VAS) with terminal descriptors of $(0=$ no pain; $10=$ maximal pain $)$. Self-reported

Table I: Outcome measures used in the randomised controlled trial

\begin{tabular}{|c|c|}
\hline Primary Outcome & Measurement \\
\hline Usual pain on movement in the previous week & $\begin{array}{l}0-100 \mathrm{~mm} \text { visual analogue scale (VAS) with terminal descriptors: } 0=\text { no } \\
\text { pain; } 10=\text { maximal pain }\end{array}$ \\
\hline Usual pain during nominated aggravating activity in the previous week & $\begin{array}{l}0-100 \mathrm{~mm} \text { visual analogue scale (VAS) with terminal descriptors: } 0=\text { no } \\
\text { pain; } 10=\text { maximal pain }\end{array}$ \\
\hline Self reported difficulty with physical function & $\begin{array}{l}\text { Physical Function subscale of the Western and Onatario MacMasters } \\
\text { University (WOMAC) Osteoarthritis Index (Likert version) }\end{array}$ \\
\hline Secondary Outcomes & Measurement \\
\hline \multicolumn{2}{|l|}{ Symptoms } \\
\hline Pain and stiffness & Pain and Stiffness subscales of the WOMAC \\
\hline Perceived global effect score & $\begin{array}{l}5 \text { point ordinal scale } \\
\text { (I-much improved; } 2 \text {-improved. 3-same; 4-worse; 5-much worse) }\end{array}$ \\
\hline Sports and recreation function & $\begin{array}{l}\text { Sports and recreation function dimension of the Knee Injury and } \\
\text { Osteoarthritis Score (KOOS) }\end{array}$ \\
\hline Symptoms & Symptoms dimension of the KOOS \\
\hline Knee related quality-of-life & Knee related quality-of-life dimension of the KOOS \\
\hline \multicolumn{2}{|l|}{ Function } \\
\hline Lower extremity functional performance & $\begin{array}{l}\text { One-leg rise test - maximum number of one-leg rises from sitting on a } 0.48 \\
\mathrm{~m} \text { stool }\end{array}$ \\
\hline Stair ambulation performance & Timed stair ascent and descent \\
\hline Standing balance & $\begin{array}{l}\text { Step test - number of times can step foot up and down off } 15 \mathrm{~cm} \text { step in } 15 \\
\mathrm{~s}\end{array}$ \\
\hline Other Outcomes & Measurement \\
\hline Physical activity levels & Physical Activity Scale for the Elderly (PASE) \\
\hline Adherence (physiotherapy group only) & $\begin{array}{l}\text { Number of physiotherapy visits Completion of home exercises via log- } \\
\text { book }\end{array}$ \\
\hline Knee-related medication use & Log-book \\
\hline Adverse effects & Log-book and open probe questioning \\
\hline
\end{tabular}


difficulty with physical function will be assessed using the physical function subscale of the Likert version of the WOMAC [25]. This disease-specific measure is reliable, valid and responsive and comprises 17 items, using a 5point scale to score each, where higher scores indicate worse symptoms.

\section{Secondary outcome measures}

Pain and stiffness will be assessed using the relevant subscales of the WOMAC [25]. Participants will rate their perceived overall change in symptoms following treatment on a 5 point ordinal scale: 1 -much improved, 2 -improved, 3-no change, 4-worse, 5-much worse, giving a perceived global effect score. Sports and recreation function, symptoms and knee-related quality-of-life will be assessed using the relevant dimensions of the Knee Injury and Osteoarthritis Outcome Score (KOOS) [26].

Objective measures of function will include the one-leg rise test, a timed stair ambulation test and the step-test. The one-leg rise test is the maximum number of one-leg rises the participant can perform from sitting on a stool. The participant must hold their non-test leg out straight and cannot use their arms for assistance. The number of rises that the participant can complete will be recorded. This test is a measure of lower extremity functional performance that has been found to predict the development of radiographic knee OA in middle aged people with chronic knee pain [27]. The timed stair ambulation task involves the participant ascending and descending a set of nine standard steps at their usual pace and the total time taken recorded, with longer time taken indicating poorer physical function [28]. The step-test is a functional, dynamic test of standing balance, where the participants stands on one leg in front of a $15 \mathrm{~cm}$ step, and places the opposite foot on and off the step as quickly as possible over $15 \mathrm{sec}-$ onds. The total number of successful steps are recorded, with higher scores indicating better balance [29].

\section{Other measures}

Disease severity of the TFJ from weight bearing anteroposterior knee x-rays taken at screening will be determined using the Kellgren and Lawrence grading system [23] where $0=$ normal $; 1=$ possible osteophytes; $2=$ minimal osteophytes and possible joint space narrowing; 3 = moderate osteophytes, some narrowing and possible sclerosis and; 4 = large osteophytes, definite narrowing and severe sclerosis. PFJ OA will be assessed from a skyline x-ray using a radiographic atlas [22]. The medial and lateral PFJ compartments will each be scored separately for the presence of osteophytes and joint space narrowing where $0=$ normal; $1=$ mild or $1-33 \%$ abnormal; $2=$ moderate or $34-66 \%$ abnormal and; $3=$ severe or $67-100 \%$ abnormal. Co-interventions, adherence and adverse effects will also be recorded. Participants will be asked to refrain from other forms of OA treatment, but stable drug doses will be permitted. Physiotherapists will record attendance, details of treatment progression (physiotherapy group) and adverse events. Participants will record adherence with home exercises (physiotherapy group), adverse events and any cointerventions, including knee-related medication use in a log-book.

\section{Interventions}

Each participant will be treated by an experienced and registered physiotherapist. Treating practitioners will be trained and proficient in both of the interventions (physiotherapy and education control). Each treatment will be delivered in 8 sessions over 12 weeks (once per week for four weeks, then once every two weeks for 8 weeks). Reasonable costs associated with treatments will be met by the project.

\section{Physiotherapy Treatment}

The physiotherapy treatment will be similar to that employed in our previous RCTs for patellofemoral pain in younger people $[19,20]$. Treatment will consist of (i) functional retraining exercises for the quadriceps muscle; (ii) quadriceps and hip muscle strengthening; (iii) patellar taping; (iv) manual PFJ and soft tissue mobilisation; and (v) OA education. The treatment will be tailored according to each participant's clinical presentation (eg strength, pain severity, swelling) as well as the presence of co-morbidities (eg back and hip pain or pathology), and will be progressed based on individual response to exercise load, thus optimising treatment effects. Exercises will be taught and supervised by the physiotherapist during each visit. A home exercise program will be prescribed, to be performed independently at home 4 times per week. An exercise manual for participants will be produced, with clear instructions and diagrams to ensure correct and safe performance of exercise. Specific aspects of the treatment are outlined in Table 2 and will include:

(i) Functional retraining exercises for the quadriceps muscle. The muscle retraining is designed to enhance the coordination (magnitude and onset timing) of the medial quadriceps, relative to the lateral utilising biofeedback within the sessions. In order to accommodate a patient group with heterogeneous symptoms, the functional retraining exercises may be performed statically and/or dynamically during various functional activities (eg step up, step down, sit to stand).

(ii) Quadriceps and hip abduction strengthening. The exercise selection will be based on baseline capacity of the individual and then progressed, based on response to exercise load, thus maximising the training effects. Resistance will be provided by weights, rubber tubing and/or body weight. 
Table 2: Physiotherapy treatment components

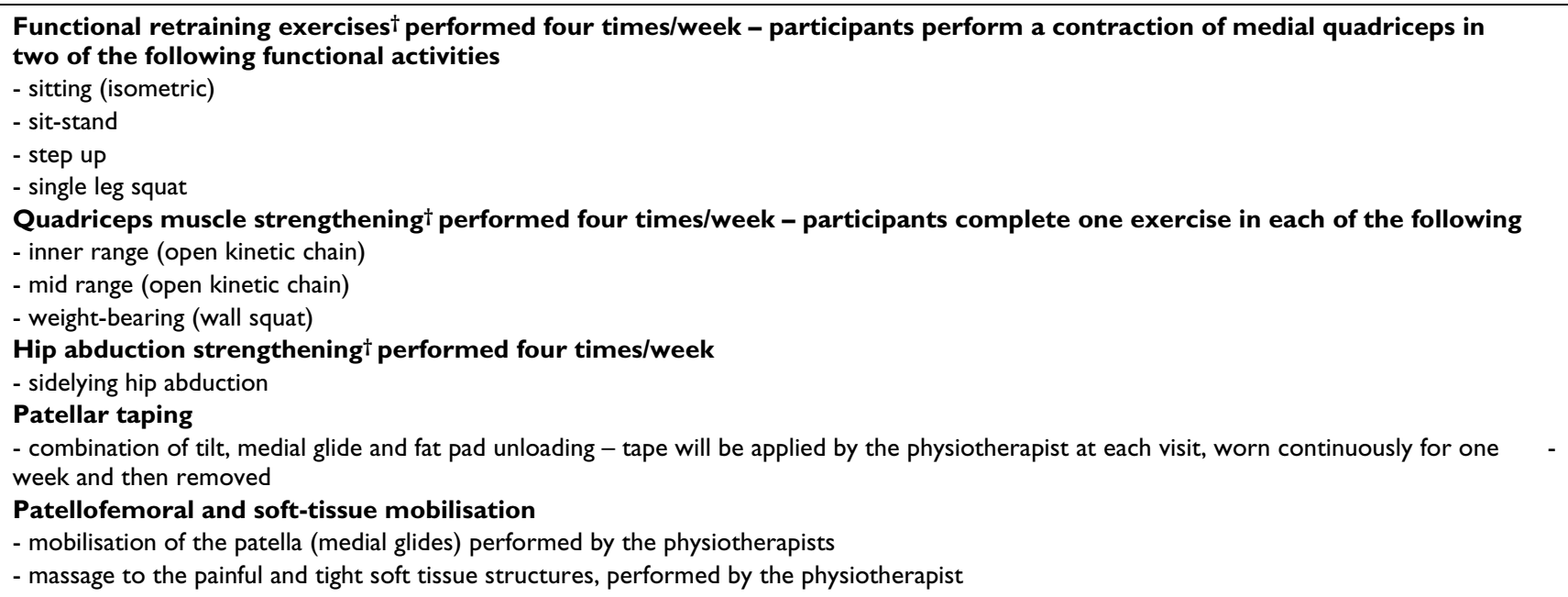

tExact exercise and its number of repetitions will be determined by the physiotherapist from a schedule of permissible exercises based on each participant's clinical presentation (eg strength, pain severity, swelling), presence of comorbidities (eg back and hip pain or pathology) and will be progressed based on individual response to exercise load

(iii) Patellar taping to reduce pain using the same standardised protocol as per our previous knee OA research $[30,31]$. The tape will be applied by the physiotherapist at each visit, worn continuously for one week and then removed.

(iv) Manual PFJ and soft tissue mobilisation, comprising medial patellar glides and massage to the lateral soft tissue structures, performed by the physiotherapist.

(v) OA education covering topics such as exercise, diet, weight loss etc.

Following cessation of supervised physiotherapy sessions at 12 weeks, participants will be instructed to continue with a home exercise program. Adherence to the program will be monitored from the diary recordings of exercise completions.

\section{Physiotherapy Education Control}

In order to control for the psychosocial contact inherent with the physiotherapy treatment, participants allocated to the control group will attend individualised OA education sessions covering topics such as exercise, diet, weight loss, etc, provided by the physiotherapist with the same frequency as the physiotherapy sessions.

\section{Data quality and management}

Strategies employed to ensure data quality include: (i) training of assessors and physiotherapists; (ii) assessment of procedural quality; (iii) random checks by investigators of adherence to study protocols; and (iv) random checks of forms for completeness and data for accuracy. All anal- yses will be conducted on an intention-to-treat basis. The primary outcomes measured at 12 weeks and 9 months will be analysed using linear mixed regression models, including their respective baseline scores as a covariate, subjects as a random effect, treatment condition as a fixed factor and the covariate by treatment interaction. Participant characteristics (eg; gender, radiographic severity of $\mathrm{TFJ}$ and PFJ OA) will also be included as covariates. Regression diagnostics will be used to check for normality of the measures and homogeneity of variance, where appropriate. Comparisons between group means will be performed using Bonferroni or Newman Keuls range tests. An alpha level of 0.05 will be used. Calculation of the number needed to treat index will be performed to facilitate the development of clinical guidelines.

\section{Discussion and Conclusion}

PFJ OA is emerging as a distinct clinical entity that is common, is associated with considerable pain and disability, and is an important and novel area of research, since little is known about the optimal management of this condition. This study uses a single-blind RCT design to investigate whether a multimodal physiotherapy treatment, targeted to the PFJ, is more effective in reducing pain and improving physical function than a physiotherapy education control intervention in people with PFJ OA. As a secondary aim, it will evaluate whether the targeted physiotherapy treatment results in greater perceived improvement, self-reported stiffness, pain, sport and recreational function, symptoms and knee-related quality of life, as well as performance on functionally relevant tasks (one-leg rises, timed stair ambulation, and step-test) than the physiotherapy education control intervention. 
In contrast to OA primarily affecting the TFJ, comparatively little known about the features or impairments associated with OA of the PFJ, and hence designing a targeted intervention is challenging. Thus, we have chosen to investigate a physiotherapy intervention that is largely based on a program that we have previously found to be successful in younger people with PFJ pain (patellofemoral pain syndrome) $[19,20]$. Components of this targeted intervention include: (i) functional retraining of the quadriceps muscle; (ii) quadriceps and hip muscle strengthening; (iii) patellar taping; (iv) manual PFJ and soft tissue mobilisation; and (v) OA education. This intervention is currently considered to be "best-practice" in the management of PFJ pain, and is increasingly being employed clinically in the management of people with PFJ OA.

An impairment that has been the subject of recent evaluation in participants with generalised knee OA is patellar malalignment. Patellar malalignment is typically exhibited as lateral patellar tilt, displacement or subluxation and may be important in PFJ OA by reducing and lateralising the PFJ contact area [32], thus increasing stress in this compartment. In people with knee OA, PFJ malalignment has been shown to be associated with indices of OA (joint space narrowing and loss of cartilage thickness) $[33,34]$ as well as progression of OA (joint space narrowing) [35] in the PFJ compartment and increased functional impairment [36]. Thus, PFJ malalignment is a key feature of PFJ OA that could be amenable to a targeted intervention such as physiotherapy. This supports the inclusion of patellar tape in our targeted treatment, since it has the potential to reduce patellar malalignment [3739] and we have already shown that patellar tape can reduce knee pain in generalised knee OA populations $[30,31]$. Other treatment modalities (eg PFJ and soft tissue mobilisations), may assist in the treatment of PFJ pain and malalignment in this patient population.

The balance of medial and lateral quadriceps activity is essential to maintain PFJ alignment. Experimental studies confirm that reduced or delayed medial quadriceps activity (relative to the lateral quadriceps) increases lateral patellar malalignment, leading to areas of heightened contact stress across the lateral PFJ compartment $[40,41]$. Thus, the balance of muscle activation between the medial and lateral quadriceps may be important in PFJ disease. In our studies of younger people with PFJ pain $[42,43]$, we have observed a temporal delay in medial quadriceps activity. Thus, it is likely that individuals with PFJ OA may require a specific retraining program designed to restore balanced quadriceps activity.

While the role of hip muscle function in PFJ OA has not been investigated, there is increasing evidence that hip muscle function is impaired (reduced strength [44], delayed hip muscle activity [45]; and altered hip movements during ambulation [46]) in other PFJ conditions. These studies indicate that hip abduction is particularly relevant in patients with PFJ pain and hence, this study is focusing on strengthening hip abduction. Furthermore, the inclusion of a hip abduction strengthening program in this study reflects contemporary clinical practice.

While the main goal of treatment for OA is to reduce pain and disability, it is not known how non-pharmacological interventions achieve this goal; such is the complex multifactorial nature of OA pain. Our intervention is based on reversing the compartment-specific impairments likely to be associated with PFJ OA. Furthermore, this intervention builds on our previous studies, which have established that: (i) taping the patella medially reduces pain and disability associated with non-specific knee OA [30,31,47] and may reduce PFJ malalignment [48] and (ii) a quadriceps retraining program can reduce pain and disability, as well as restore quadriceps muscle activation patterns in younger people with PFJ pain $[19,49,50]$. Our unique RCT is targeting PFJ OA, an important sub-group of knee $\mathrm{OA}$, with a specifically designed intervention. The project's outcome will influence knee OA rehabilitation, thus reducing the personal and societal burden of this increasing public health problem.

\section{Competing interests}

The authors declare that they have no competing interests.

\section{Authors' contributions}

$\mathrm{KC}, \mathrm{RH}, \mathrm{BV}, \mathrm{MP}, \mathrm{AS}$ attained the project funding. $\mathrm{KC}, \mathrm{RH}$, $\mathrm{BV}$ conceived and designed the trial protocol. All authors contributed to the manuscript and have read and approved the final manuscript.

\section{Acknowledgements}

This trial is funded by the National Health and Medical Research Council Project Grant (Project \# 508966).

\section{References}

I. March L, Bagga H: Epidemiology of osteoarthritis in Australia. Medical Journal of Australia 2004, 180:S6-SI0.

2. Access Economics Pty Ltd: Painful Realities: The economic impact of arthritis in Australia. Arthritis Australia 2007.

3. Szebenyi B, Hollander A, Dieppe P, Quilty B, Duddy J, Clarke S, J K: Associations between pain, function, and radiographic features in osteoarthritis of the knee. Arthritis \& Rheumatism 2006, 54:230-5.

4. Duncan R, Hay E, Saklatvala J, Croft P: Prevalence of radiographic osteoarthritis: It all depends on your point of view. Rheumatology 2006, 45:757-60.

5. Iwano T, Kurosawa H, Tokuyama H, Hoshikawa Y: Roentographic and clinical findings of patellofemoral arthritis. Clinical Orthopaedics \& Related Research 1990, 252:190-7.

6. Elahi S, Cahue S, Felson D, Engelman L, Sharma L: The association between varus-valgus alignment and patellofemoral alignment. Arthritis \& Rheumatism 2000, 43:9|4-8.

7. Mazzuca EM, Brandt KD, Katz JN, Ding Y, Lane K, Buckwater K: Risk factors for progression of tibiofemoral osteoarthritis: an 
analysis based on fluroscopically standardised knee radiography. Annals of the Rheumatic Diseases 2006, 65:5I5-9.

8. Hinman RS, Crossley KM: Patellofemoral osteoarthritis: an important subgroup of knee osteoarthritis. Rheumatology 2007, 46:1057-62

9. Kornaat PR, Bloem LL, Ceulemans RYT, Riyazi N, Rosendaal FR, Nelissen RG, Carter WO, Le Graverand MPH, Kloppenburg M: Osteoarthritis of the knee: Association between clinical features and MR imaging findings. Radiology 2006, 239:8II-7.

10. Hunter DJ, March L, Sambrook PN: The association of cartilage volume with knee pain. Osteoarthritis \& Cartilage 2003, I I:725-9.

II. Boegard T, Rudling O, Petersson IF, Sanfridsson J, Saxne T, Svensson $B$, Jonsson $\mathrm{K}$ : Joint-space width in the axial view of the patellofemoral joint. Definitions and comparisons with MR imaging. Acta Radiologica 1998, 39:24-31.

12. Ciccutini F, Baker D, hart J, Spector T: Association of pain with radiological changes in different compartments and views of the knee joint. Osteoarthritis \& Cartilage 1996, 4:143-7.

13. Lanyon P, O'Reilly S, Jones A, Doherty M: Radiographic assessment of symptomatic knee osteoarthritis in the community: definitions and normal joint space. Annals of the Rheumatic Diseases 1998, 57:595-601.

14. Tallon D, Chard J, Dieppe P: Relation between agendas of the research community and the research consumer. Lancet 2000, 355:2037-40.

15. Jordan KM, Arden NK, Doherty M, Bannwarth B, Bijlsma JWJ, Dieppe $P$, Gunther K, Hauselmann H, Herrero-Beaumont G, Kaklamanis P, Lohmander S, Leeb B, Lequesne M, Mazieres B, Martin-Mola E, Pavelka K, Pendleton A, Punzi L, Serni U, Swoboda B, Verbruggen G, Zimmerman-Gorska I, Dougados M: EULAR Recommendations 2003: an evidence based approach to the management of knee osteoarthritis: Report of a Task Force of the Standing Committee for International Clinical Studies Including Therapeutic Trials (ESCISIT). Annals of the Rheumatic Diseases 2003, 62: I| I45-55

16. American College of Rheumatology: Recommendations for the medical management of osteoarthritis of the hip and knee. 2000 update. Arthritis \& Rheumatism 2000, 43:1905-I5.

17. Bennell KL, Hinman RS: Exercise as a treatment for osteoarthritis. Current Opinion in Rheumatology 2005, I7:634-40.

18. Quilty B, Tucker M, Campbell R, Dieppe P: Physiotherapy, including quadriceps exercises and patellar taping, for knee osteoarthritis with predominant patellofemoral involvement: randomized controlled trial. Journal of Rheumatology 2003, 30:1311-7.

19. Crossley K, Bennell K, Green S, Cowan S, McConnell J: Physical therapy for patellofemoral pain: A randomised, doubleblind, placebo controlled trial. American Journal of Sports Medicine 2002, 30:857-65.

20. Vicenzino B, Collins N, Crossley KM, Beller E, Darnell R, McPoil T: Foot orthoses and physiotherapy in the treatment of patellofemoral pain syndrome: A randomised clinical trial. $B M C$ Musculoskeletal Disorders 2008, 9:.

21. Altman DG: Better reporting of randomised controlled trials: the CONSORT statement. British Medical Journal 1996, 313:570-I.

22. Altman RD, Hochberg M, Murphy WA, Wolfe F, Lequesne M: Atlas of individual radiographic features in osteoarthritis. Osteoarthritis \& Cartilage 1995, 3:3-70.

23. Kellgren JH, Jeffrey MR: The Epidemiology of Chronic Rheumatism: Atlas of Standard Radiographs. In Oxford, Blackwell Scientific; 1963 .

24. Tubach F, Ravaud P, Baron G, Falissard B, Logeart I, Bellamy N, Bombardier C, Felson D, Hochberg M, Heijde D van der, M D: Evaluation of clinically relevant changes in patient reported outcomes in knee and hip osteoarthritis: the minimal clinically important improvement. Annals of the Rheumatic Diseases 2005, 64:29-33.

25. Bellamy N, Buchanon WW, Goldsmith $\mathrm{CH}$, Campbell J, Stitt L: Validation study of WOMAC: a health status instrument for measuring clinically-important patient-relevant outcomes following total hip or knee arthroplasty in osteoarthritis. Journal of Rheumatology 1988, 15:1833-40.

26. Roos EM, Roos HP, Lohmander LS, Ekdahl C, Beynnon BD: Knee Injury and Osteoarthritis Outcome Score (KOOS) - devel- opment of a self-administered outcome measure. J Orthop Sports Phys Ther 1998, 28(2):88-96.

27. Thorstensson CA, Petersson IF, Jacobsson LTH, Boegard TL, Roos EM: Reduced functional performance in the lower extremity predicted radiographic knee osteoarthritis five years later. Annals of the Rheumatic Diseases 2004, 63:402-7.

28. Kennedy DM, Stratford PW, Wessel J, Gollish JD, Penney D: Assessing stability and change of four performance measures: $a$ longitudinal study evaluating outcome following total hip and knee arthroplasty. Bmc Musculoskeletal Disorders 2005, 6:.

29. Hinman RS, Bennell KL, Metcalf BR, Crossley KM: Balance impairments in individuals with symptomatic knee osteoartritis: $A$ comparison with matched controls using clinical tests. Rheumatology 2002, 41 : I 388-94.

30. Hinman R, Crossley KM, McConnell J, Bennell K: Efficacy of knee tape in the management of knee osteoarthritis: blinded, randomised controlled trial. British Medical Journal 2003, 327:135-8.

31. Hinman RS, Bennell KL, Crossley KM, McConnell J: Immediate effects of adhesive tape on pain and disability in individuals with knee osteoarthritis. Rheumatology 2003, 42:865-9.

32. Hinterwimmer S, Gotthardt M, von Eisenhart-Rothe R, Sauerland $S$, Siebert M, Vogl T, Eckstein F, Graichen $H$ : In vivo contact areas of the knee in patients with patellar subluxation. Journal of Biomechanics 2005, 38:2095-I0I.

33. Kalichman L, Zhang Y, Niu J, Goggins J, Gale D, Felson DT, Hunter D: The association between patellar alignment and patellofemoral joint osteoarthritis features - an MRI study. Rheumatology 2007, 46: 1303-8.

34. Kalichman L, Zhang YQ, Niu JB, Goggins J, Gale D, Zhu YY, Felson $D T$, Hunter DJ: The association between patellar alignment on magnetic resonance imaging and radiographic manifestations of knee osteoarthritis. Arthritis Research \& Therapy 2007, 9:

35. Hunter DJ, Zhang YQ, Niu JB, Felson DT, Kwoh K, Newman A, Kritchevsky S, Harris T, Carbone L, Nevitt M: Patella malalignment, pain and patellofemoral progression: the Health ABC Study. Osteoarthritis and Cartilage 2007, I 5: I | 20-7.

36. Kalichman L, Zhu Y, Zhang Y, Niu J, Gale D, Felson DT, Hunter D: The association between patella alignment and knee pain and function: an MRI study in persons with symptomatic knee osteoarthritis. Osteoarthritis and Cartilage 2007, I 5: I235-40.

37. Worrell T, Ingersoll CD, Bockrath-Pugliese K, Minis P: Effect of patellar taping and bracing on patellar position as determined by MRI in patients with patellofemoral pain. Journal of Athletic Training 1998, 33:16-20.

38. Larsen B, Adreasen E, Urfer A Mickelson MR, Newhouse KE: Patellar taping: a radiographic examination of the medial glide technique. American Journal of Sports Medicine 1995, 23:465-7I.

39. Pfeiffer RP, DeBeliso M, Shea KG, Kelley L, Irmischer B, Harris C Kinematic MRI assessment of McConnell taping before and after exercise. American Journal of Sports Medicine 2004, 32:62I-8.

40. Lin F, Wang G, Koh JL, Hendrix RW, Zhang L-Q: In vivo and noninvasive three-dimensional patellar tracking induced by individual heads of quadriceps. Medicine \& Science in Sport \& Exercise 2004, 36:93-101.

41. Neptune RR, Wright IC, bogert AJ Van den: The influence of orthotic devices and vastus medialis strength and timing on patellofemoral loads during running. Clinical Biomechanics 2000, 15:6II-8.

42. Cowan SM, Bennell KL, Hodges PW, Crossley KM, McConnell ! Delayed onset of electromyographic activity of vastus medialis obliquus relative to vastus lateralis in subjects with patellofemoral pain syndrome. Archives of Physical Medicine and Rehabilitation 200I, 82: 183-9.

43. Cowan SM, Hodges PW, Bennell KL, Crossley KM: Altered vasti recruitment when people with patellofemoral pain syndrome complete a postural task. Archives of Physical Medicine and Rehabilitation 2002, 83:989-95.

44. Ireland ML, Wilson JD, Ballantyne BT, McClay Davis I: Hip strength in females with and without patellofemoral pain. Journal of Orthopaedic and Sports Physical Therapy 2003, 33:67I-6.

45. Brindle TJ, Mattacola C, McCrory J: Electromyographic changes in the gluteus medius during stair ascent and descent in subjects with anterior knee pain. Knee Surgery Sports Traumatology Arthroscopy 2003, II:244-5I.

46. Willson J, Davis IS: Lower extremity mechanics of females with and without patellofemoral pain across activities with pro- 
gressively greater demands. Clinical Biomechanics 2008, 23:203-II.

47. Warden Sj, Hinman RS, Watson MAJ, Avin KG, Bialocerkowski AE, Crossley KM: Patellar taping and bracing for the treatment of chronic knee pain: a systematic review and meta-analysis. Arthritis \& Rheumatism (Arthritis Care \& Research) 2008, 59:78-83.

48. Crossley K, Bennell K, Green S, McConnell J: A systematic review of physical interventions for patellofemoral pain syndrome. Clinical Journal of Sports Medicine 2001, I I:103-10.

49. Cowan SM, Bennell KL, Crossley KM, Hodges PW, McConnell J: Physiotherapy treatment alters the recruitment of the vasti in patellofemoral pain syndrome. Medicine and Science in Sports and Exercise 2002, 34:1879-85.

50. Cowan SM, Bennell KL, Hodges PW, Crossley KM, McConnell J: Simultaneous feedforward recruitment of the vastii in untrained postural tasks can be restored by specific training. Journal of Orthopaedic Research 2003, 21:553-8.

\section{Pre-publication history}

The pre-publication history for this paper can be accessed here:

http://www.biomedcentral.com/1471-2474/9/122/pre

pub

Publish with Bio Med Central and every scientist can read your work free of charge

"BioMed Central will be the most significant development for disseminating the results of biomedical research in our lifetime. "

Sir Paul Nurse, Cancer Research UK

Your research papers will be:

- available free of charge to the entire biomedical community

- peer reviewed and published immediately upon acceptance

- cited in PubMed and archived on PubMed Central

- yours - you keep the copyright

Submit your manuscript here:

http://www.biomedcentral.com/info/publishing_adv.asp 